\title{
Pisot-K Elements in the Field of Formal Power Series over Finite Field
}

\section{Kthiri $\mathrm{H}^{*}$}

Department of Mathematics, University of Sfax, Tunisa

\begin{abstract}
In this paper, we will give a criteria of irreducible polynomials over $\mathbb{F}_{q}[X]$ where $\mathbb{F}_{q}$ is a finite field. We will present an estimation for the number of the Pisot-k power formal series, precisely we will give their degrees and their logarithmic heights.
\end{abstract}

Keywords: Formal power series; Irreducible polynomials; Pisot-2 series. $|z|<1$

\section{Introduction}

In 2007, Gabriel Ponce, Melanie Ruiz, Emily McLeod Schnitger and Noah Simon have discuss certain sets of algebraic integers related to Pisot numbers (An integer algebraic number $\alpha>1$ is called a Pisot number if all its conjugates, different from $\alpha$ lie in the open disc $|z|<1$ on the complex plane) and Salem number (An integer algebraic number $\alpha>1$ is called a Salem number if all its conjugates, different from $\alpha$ lie in the disc $|z|<1$ and it has at least one conjugate having modulus equal to 1) Also their properties.

Much is known about Pisot numbers, for example, if the integer coefficients of the minimal polynomial of $\alpha P(z)=z^{d}+q_{d-1} z^{d-1}+\cdots+q_{0}$, satisfy $1+\sum_{i=0}^{d-1} q_{i}<0$ and $P(x)=x^{d}-a_{d-1} x^{d-1}-\cdots-a_{0}$. then a is a Pisot number. Also, for $\alpha \in s$ it is known that $\alpha^{n} \in s$ One important theorem about Pisot numbers is that the set $S$ is closed and there are many known ways to construct them.

Let $\alpha$ be a root dominate of polynomial $P(x)=x^{d}-a_{d-1} x^{d-1}-\cdots-a_{0}$. [1].

$a_{d-1} \geq a_{d-2} \geq \cdots \geq a_{0}>0$ where $a_{\mathrm{i}} \in \mathbb{N}^{*}$ then $\alpha$ is a Pisot number. If $a_{d-1}>\sum_{i=0}^{d-2}\left|a_{i}\right|+1$ where $a_{\mathrm{i}} \in \mathbb{Z}$ and $a_{0} \neq 0$ then $\alpha$ is a Pisot number.

If $a_{d-1}>\sum_{i=2}^{d} a_{i}, a_{i} \geq 0$ and $a_{d} \neq 0$, then $\alpha$ is a Pisot number [2].

In comparison, little is known about Salem numbers, and their construction is difficult. There are still many open questions about Salem numbers, including determining the infest of the set.

Definition 1.1: The reciprocal polynomial of a polynomial $P$ is defined by $P^{*}(z)=z^{\operatorname{deg} P} P\left(\frac{1}{z}\right)$. A polynomial is called reciprocal if $P=P^{*}$ In general, we will denote the reciprocal polynomial of $P$ by $Q$.

The minimal polynomial of a Salem number will always be reciprocal, and thus, there are no Salem numbers with odd degree or degree less than 4. Constructing Salem numbers is much more difficult than Pisot numbers. For instance, graph theory can be used to construct some but not nearly all. The smallest Salem number is still not known, though it is conjectured to be largest root of $1+z-z^{3}-z^{4}-z^{6}-z^{7}+z^{9}+z^{10}$.

They have defined new sets of generalized Pisot numbers and they have concerned with the arithmetic properties and limit points of one of these sets, Pisot-2 pairs (A pair of real distinct algebraic conjugates $\left(\alpha_{1} \alpha_{2} \ldots, \alpha_{k}\right)$ is called a Pisot-k uplet or o(k)-Pisot number if $\alpha_{1}, \alpha_{2} \ldots, \alpha_{k}$ are greater than 1 and all remaining conjugates have modulus strictly less than 1) We denote the set of Pisot-k pairs by $S_{k}$ For this set they have obtained some results analogous to those known about Pisot numbers. $\left(\alpha_{1}, \alpha_{2}\right) \in S_{2}$

Proposition 1.1: If $\left(\alpha_{1}, \alpha_{2}\right) \in S_{2}$ then $\left(\alpha_{1}^{n}, \alpha_{2}^{n}\right) \in S_{2}$ for all $n \in \mathbb{N}$.
Proposition 1.2: If $\left(\alpha_{1} \alpha_{2}\right) \in S_{2}$ then $\left\{\alpha_{1}^{n}+\alpha_{2}^{n}\right\} \rightarrow 0$ for all $n \in \mathbb{N}$

Proposition 1.3: If $\left(\alpha_{1}, \alpha_{2}\right) \in S_{2}$ with minimal polynomial, $P \in \mathbb{Q}[X]$ of degree 3 and $P(0)=1$ then $\alpha_{1}, \alpha_{2} \in S_{2}$

Theorem 1.4: The limit points of $S_{2}$ lie either in $S_{2} S \times$ or $\mathbb{R} \times S$

A great deal is known about this set. Then, they have discussed another set, Pisot (o)-2 numbers, and its connections to Salem numbers, including a relationship with the infemum of Salem numbers. Finally, They have giving arithmetic properties of these Pisot (o)-2 numbers. In this paper, we consider an analogue of this concept in algebraic function over finite fields.

Recall that in 1962 Batemen and Duquettes introduced and characterized the elements of Pisot and Salem in the field of formal power series [3].

Theorem 1.5: [4] Let. $w \in \mathbb{F}_{q}\left(\left(X^{-1}\right)\right)$ an algebraic integer over $\mathbb{F}_{q}[X]$ and its minimal polynomial be

$$
P(y)=y^{n}+A_{n-1} y^{n-1}+\cdots+A_{0}, A_{i} \in \mathbb{F}_{q}[X]
$$

Then $w$ is a Pisot (respectively Salem) series if and only if $\left|A_{n-1}\right|>\max _{i \neq n-1}\left|A_{i}\right|\left(\right.$ respectively $\left.\left|A_{1}\right|=\max _{i \neq n-1}\left|A_{i}\right|\right)$.

Chandoul, Jellali and Mkaouar have improve the Theorem of Bateman and Duquette [4] on $\mathbb{F}_{q}\left(\left(X^{-1}\right)\right)$ and this while establishing the same result with weaker hypotheses [4].

Theorem 1.6: [2] Let $w \in \mathbb{F}_{q}\left(\left(X^{-1}\right)\right)$ be Pisot (respectively Salem) series if and only if $w$ is a root of polynomial

$P(y)=y^{n}+A_{n-1} y^{n-1}+\cdots+A_{0}, A_{i} \in \mathbb{F}_{q}[X]$

where $\left|A_{n-1}\right|>\max _{i \neq n-1}\left|A_{i}\right|\left(\right.$ respectively $\left.\left|A_{n-1}\right|=\max _{i \neq n-1}\left|A_{i}\right|\right)$.

In the same setting and on the field of the real, Brauer, gave a criteria of irreducibility on $\mathbb{Q}[5]$

Theorem 1.7: If $a_{1}, a_{2}, \cdots, a_{n-1}$ are of the integer such that $a_{n-1} \geq a_{n-2} \geq \cdots \geq a_{0}>0$, thenthepolynomial $x^{n}-a_{n-1} x^{n-1}-a_{n-2} x^{n-2}-\cdots-a_{0}$ is irreducible on $\mathbb{Q}$

*Corresponding author: Kthiri $\mathrm{H}$, Department of Mathematics, University of Sfax Tunisa, Tel: +216 74242 951; E-mail: hassenkthirigmail.com

Received June 15, 2016; Accepted August 22, 2016; Published August 25, 2016

Citation: Kthiri H (2016) Pisot-K Elements in the Field of Formal Power Series over Finite Field. J Phys Math 7: 194. doi: 10.4172/2090-0902.1000194

Copyright: (c) 2016 Kthiri $\mathrm{H}$. This is an open-access article distributed under the terms of the Creative Commons Attribution License, which permits unrestricted use, distribution, and reproduction in any medium, provided the original author and source are credited. 
Chandoul, Jellali and Mkaouar are constructs the analog of the theorem of Brauer in the case of the polynomials to coefficients in $[X][4]$.

Theorem 1.8: Let $\Lambda(Y)=Y^{d}+\lambda_{d-1} Y^{d-1}+\cdots+\lambda_{0}$ where $\lambda_{i} \in \mathbb{F}_{q}[X], \lambda_{0} \neq 0$ and $\operatorname{deg} \lambda_{d-1}>\operatorname{deg} \lambda_{i}$, for all $i \neq d-1$ then $\Lambda$ is irreducible on $\mathbb{F}_{q}[X]$.

This last theorem permits to give a new evaluation to calculate the number of elements of Pisot on $\mathbb{F}_{q}\left(\left(X^{-1}\right)\right)$ being given their $n$ degrees and their logarithmic heights $h$ This evaluation is illustrated in the following result:

$$
\mathcal{P}(n, h)=(q-1)\left(q^{h}-1\right) q^{(n-1) h} .
$$

The smallest Pisot number, in the real case, is the only real root of the polynomial $x^{3}-x-1$ known as the plastic number or number of money (approximately 1,324718 But, as we have said, the smallest accumulation point of all Pisot numbers is the golden number. Chandoul, Jellali and Mkaouar are prove in [5] that the minimal polynomial of the smallest Pisot element (SPE) of degree $n$ is $P(Y)=Y^{n}-\alpha X Y^{n-1}-\alpha^{n}$ where $\alpha$ is the least element of $F_{q} \backslash\{0\}$ Moreover, the sequence $\left(w_{n}\right)_{n 1}$ is decreasing one and converge to $\alpha X$ In the present paper we give generalized Pisot and Salem series in the field of formal power series over finite field. $P(Y)=$ $Y^{n}-\alpha X Y^{n-1}-\alpha^{n}$

The paper is organized as follows. In this section, we give some preliminary definitions, we define Pisot numbers and Salem numbers. We give the well known properties of its. In section 2 we introduce the field of formal power series over finite field, we define a totally order, the lexicographic order, over a field of formal power series with coefficients in a finite field. In section 3 We give the arithmetic Properties of Pisot (o)-k series and the criteria of irreducible of this series.

\section{of Formal Series $\mathbb{F}_{q}\left(\left(X^{-1}\right)\right)$}

Let be $\mathbb{F}_{q}$ a finite field of $q$ elements, $\mathbb{F}_{q}[X]$ the ring of polynomials with coefficient $I_{q}, \mathbb{F}_{q}(X)$ the field of rational functions, $\mathbb{F}_{q}(X, \beta)$ the minimal extension of $\mathbb{F}_{q}$ containing $X$ and $\beta$ and $\mathbb{F}_{q}(x, \beta)$ the minimal ring containing $X$ and $\beta$. Let $\mathbb{F}_{q}\left(\left(X^{-1}\right)\right)$ be the field of formal power series of the form :

$$
w=\sum_{k=-\infty}^{l} w_{k} X^{k}, w_{k} \in \mathbb{F}_{q}
$$

where

$$
l=\operatorname{deg} w:= \begin{cases}\max \left\{k: w_{k} \neq 0\right\} & \text { for } w \neq 0 \\ -\infty & \text { for } w=0 .\end{cases}
$$

Define the absolute value by

$$
|w|= \begin{cases}q^{\operatorname{deg} w} & \text { for } w \neq 0 \\ 0 & \text { for } w=0 .\end{cases}
$$

Then $|$.$| is not archimedean. It fulfills the strict triangular inequality$

$$
\begin{aligned}
& |w+v| \leq \max (|w|,|v|) \quad \text { and } \\
& |w+v|=\max (|w|,|v|) \quad \text { if }|w| \neq|v| .
\end{aligned}
$$

For $f \in \mathbb{F}_{q}\left(\left(X^{-1}\right)\right)$ define the integer (polynomial) part $[w]=\sum_{k=0}^{l} w_{k} X^{k}$ where the empty sum, as usual, is defined to be zero. Therefore $f \in \mathbb{F}_{q}[X]$ and $(w-[w])$ is in the unit disk $D(0,1)$ for all $w \in \mathbb{F}_{q}\left(\left(X^{-1}\right)\right)$. As explained by Sprindzuk a non archimedean absolute value on $\mathbb{F}_{q}\left(\left(X^{-1}\right)\right)$ is defined by $|w|=q^{-s}$ It is clear that, for all $P \in \mathbb{F}_{q}[X],|P|=q^{\text {degP }}$ and, for all $Q \in \mathbb{F}_{q}$ $[X]$;such that $Q \neq 0\left|\frac{P}{Q}\right|=q^{\operatorname{deg} P-\operatorname{deg} Q}[6]$.

We know that $\mathbb{F}\left(\left(X^{-1}\right)\right)$ is complete and locally compact with respect to the metric defined by this absolute value. We denote by $\overline{\mathbb{F}}_{q}\left(\left(X^{-1}\right)\right)$ an algebraic closure of $\mathbb{F}_{q}\left(\left(X^{-1}\right)\right)$ We note that the absolute value has a unique extension to $\overline{\mathbb{F}}_{q}\left(\left(X^{-1}\right)\right)$. Abusing a little the notations, we will use the same symbol |. $\left.\right|^{q}$ for the two absolute values. For all polynomial $(P \neq 0)$

$$
P(Y)=Y^{n}+A_{1} Y^{n-1}+\cdots+A_{n}, A_{i} \in \mathbb{F}_{q}[X]
$$

We define the logarithmic hauteur $\mathbb{H}(P)$ of $P$ by

$$
\mathcal{H}(P)=\log _{q} \max _{0 \leq i \leq n}\left|A_{i}\right|=\max _{0 \leq i \leq n} \operatorname{deg}\left(A_{i}\right)
$$

Where $\log _{q} x$ designate the logarithmic function in the $q$ basis. For all element algebraic $w \in_{q}\left(\left(X^{-1}\right)\right)$ one will note by $\mathbb{H}(w)$ the logarithmic hauteur of his minimal polynomial.

Theorem 2.1: Let $w \in \mathbb{F}\left(\left(X^{-1}\right)\right)$ is of Pisot number if and only if it exist $\lambda \in_{q}\left(\left(X^{-1}\right)\right) \backslash\{0\}$ such that $\lim _{n \rightarrow+\infty}\left\{\lambda w^{n}\right\}=0$; Moreover $\lambda$ can be chosen to belong to $\mathbb{F}_{q}(X)(w)$.

Recall that $\mathbb{F}_{q}\left(\left(X^{-1}\right)\right)$ contains Pisot elements of any degree over $\mathbb{F}_{q}(X)$ Indeed, consider the polynomial $Y^{n}-a Y^{n-1}-b$ where $a, b \in \mathbb{F}_{q} \backslash\{0\}$ it can be seen easily, considering its Newton polygon, that the polynomial, which is irreducible over $(X)$ has a root $w \in \mathbb{F}\left(\left(X^{-1}\right)\right)$ such that $|w|>1$ and all of its conjugates in $\overline{\mathbb{F}}_{q}\left(\left(X^{-1}\right)\right)$ have an absolute value strictly smaller than 1.

Definition 2.1: An uplet of series algebraic conjugates $\left(w_{1} \ldots\right.$ $\left.\left.w_{k}\right) \mathbb{F}_{q}\left(\left(X^{-1}\right)\right)\right)^{k}$ is called a series of Pisot- $k$ uplet $(o(k)$-Pisot series $)$ if $w_{1}$ integer algebraic such that $\left|w_{1}\right|, \ldots .\left|w_{k}\right|$ are greater than 1 and all remaining conjugates have modulus strictly less than 1 We denote the set of Pisot-k uplet by $S_{k}^{\prime}$.

\section{Example 2.2}

1) Series of Pisot-2 of degree 2 on $\left.\mathbb{F}_{2}\left(\left(X^{-1}\right)\right)\left(w_{1} \ldots w_{k}\right) \mathbb{F}_{q}\left(\left(X^{-1}\right)\right)\right)^{k}$

Let

$P(Y)=Y^{2}+Y+X^{2}+X+1$.

Then $P$ is irreducible over $\mathbb{F}_{2}[X][Y]$ Now we show that $P$ has two roots $\left|Y_{1}\right|>1\left|Y_{2}\right|>1$ such that $\left(Y_{1}, Y_{2}\right) \in \mathbb{F}_{2}\left(\left(X^{-1}\right)\right)^{2}$ Let $Y=\sum_{i=-1}^{\infty} Y_{i} X^{-i} \in \mathbb{F}_{2}\left(\left(X^{-1}\right)\right)$ one root of $P$.Then

$$
\begin{cases}Y_{1}=X+1+\frac{1}{X^{2}}+\cdots=X+1+\frac{1}{Z_{1}} & \text { such that }\left|Z_{1}\right|>2 \\ Y_{2}=X+\frac{1}{X}+\cdots=X+\frac{1}{Z_{2}} & \text { such that }\left|Z_{2}\right|>1 .\end{cases}
$$

For $Y_{1}$ we have

$$
Y_{1}^{2}+Y_{1}+X^{2}+X+1=0 \text {. }
$$

So

$Z_{1}^{2}+Z_{1}+1=0$.

Therefore $Z_{1}$ is root of Polynomial irreducible and $Z_{1} \in \mathbb{F}_{2}\left(\left(X^{-1}\right)\right)$ So $Y_{1} \in \mathbb{F}_{2}\left(\left(X^{-1}\right)\right)$

For $Y_{2}$ we have

$Y_{2}^{3}+Y_{2}+X^{2}+X+1=0$.

Then 


$$
Z_{2}^{2}+Z_{2}+1=0
$$

Therefore $Z_{1}$ is root of Polynomial irreducible and $Z_{2} \in \mathbb{F}_{2}\left(\left(X^{-1}\right)\right)$ So $Y_{1} \in \mathbb{F}_{2}\left(\left(X^{-1}\right)\right)$

Series of Pisot-2 of degree 3 on $\mathbb{F}_{2}\left(\left(X^{-1}\right)\right)$

Let $P(Y)=Y^{3}+\left(X^{2}+1\right) Y^{2}+\left(X^{3}+X\right) Y+1$.

Then $P$ is irreducible over $\mathbb{F}_{2}[X][Y]$ because $P$ and his reciprocal polynomial $Q(Y)=Y^{3}+\left(X^{3}+X\right) Y^{2}+\left(X^{2}+1\right) Y+1$ are the same natures. However $Q$ is of type $(I)$ then it is irreducible. Therefore $P$ is also.

Now we show that $P$ has two roots $\left|Y_{1}\right|>1$ and $\left|Y_{2}\right|>1$ such that $\left(Y_{1}\right.$ $\left.Y_{2}\right) \in \mathbb{F}_{2}\left(\left(X^{-1}\right)\right)^{2}$ and $\left|Y_{3}\right|<1$ Let $Y=\sum_{i=-2}^{\infty} Y_{i} X^{-i} \in \mathbb{F}_{2}\left(\left(X^{-1}\right)\right)$ one root of $P$ Then $\left(Y_{1}, Y_{2}\right) \in \mathbb{F}_{2}\left(\left(X^{-1}\right)\right)^{2}$

$$
\begin{cases}Y_{1}=X^{2}+X+\frac{1}{X^{2}}+\cdots=X^{2}+X+\frac{1}{Z_{1}} & \text { such that }\left|Z_{1}\right|>2 \\ Y_{2}=X+1+\frac{1}{X}+\cdots=X+1+\frac{1}{Z_{2}} & \text { such that }\left|Z_{2}\right|>1 \\ Y_{3}=\frac{1}{X}+\cdots=\frac{1}{Z_{3}} & \text { such that }\left|Z_{3}\right|>1 .\end{cases}
$$

For $Y_{1}$ : we have

$Y_{1}^{3}+\left(X^{2}+1\right) Y_{1}^{2}+\left(X^{3}+X\right) Y_{1}+1=0$.

Then

$Z_{1}^{3}+\left(X^{4}+X^{3}+X^{2}+X\right) Z_{1}^{2}+(X+1) Z_{1}+1=0$.

Therefore $Z_{1}$ is of type $(I)$ and $Z_{1} \in \mathbb{F}_{2}\left(\left(X^{-1}\right)\right)$ So $Y_{1} \in \mathbb{F}_{2}\left(\left(X^{-1}\right)\right)$

For $Y_{2}$ : we have

$Y_{2}^{3}+\left(X^{2}+1\right) Y_{2}^{2}+\left(X^{3}+X\right) Y_{2}+1=0$.

Then

$Z_{1}^{3}+\left(X^{3}+X\right) Z_{1}^{2}+\left(X^{2}+1\right) Z_{1}+1=0$.

So $Z_{2}$ is of type ${ }^{(I)}$ and $Z_{2} \in \mathbb{F}_{2}\left(\left(X^{-1}\right)\right)$ So $Y_{2} \mathbb{F}_{2}\left(\left(X^{-1}\right)\right)$

For $Y_{3}$ :we have $Y_{3}=\frac{1}{Z_{3}}$ such that $\left|Z_{3}\right|>1$ Then

$Y_{3}^{3}+\left(X^{2}+1\right) Y_{3}^{2}+\left(X^{3}+X\right) Y_{3}+1=0$.

$Z_{3}^{3}+\left(X^{3}+X\right) Z_{3}^{2}+\left(X^{2}+1\right) Z_{3}+1=0$.

Thus $Z_{3}$ is of type $(I)$ and $Z_{3} \mathbb{F}_{2}\left(\left(X^{-1}\right)\right)$ So $Y_{3} \in \mathbb{F}_{2}\left(\left(X^{-1}\right)\right)$

\section{Results}

\section{Arithmetic properties of Pisot-k uplet (respectively Salem)} series

In this section we discuss some basic arithmetic properties of $S_{k}^{\prime}$. It is known that $(\alpha, \beta) \in \mathrm{S}_{2}$ if $(\alpha, \beta) \in \mathrm{S}_{2}$ then $\left(\alpha^{n}, \beta^{n}\right) \in \mathrm{S}_{2} \forall n \in \mathbb{N}$ Also, we have that $\lim _{n \rightarrow \infty}\left\{\alpha^{n}, \beta^{n}\right\} \rightarrow\{0\}$, where $\{x\}$ denotes the fractional part of $x$. Our last proposition relates a specific subset of $S_{k}^{\prime}$ to $S^{\prime}$ The algebraic closure of $\mathbb{F}_{q}\left(\left(X^{-1}\right)\right)$ will be denoted by $\overline{\mathbb{F}}_{q}\left(\left(X^{-1}\right)\right)$.

Proposition 3.1: Let $\left(w_{1}, \cdots, w_{k}\right) \in S_{k}^{\prime}\left(\right.$ respectively. $\left.\in T_{k}^{\prime}\right)$, then $\left(w_{1}^{n}, \cdots, w_{k}^{n}\right) \in S_{k}^{\prime}\left(\right.$ respectively. $\left.\in T_{k}^{\prime}\right)$ for all $n \mathbb{N}^{*}$.

Proof. Let $\left(w_{1}, \cdots, w_{k}\right) \in S_{k}^{\prime}$ (respectively. $\left.\in T_{k}^{\prime}\right)$ and $M \in \mathbb{F}_{q}[X][Y]$ the minimal polynomial of $w$ and $w=w_{1}, \ldots w_{d}$ the conjugates of $w$. Then there exist exactly $k$ conjugates $w=w_{1}, \ldots w_{k}$ of $w$ that lie outside the unit disc. Let $w_{k+1} \ldots w_{d}$ denote the other roots of $M$.

We know that the product of any two algebraic is, itself, an algebraic.
Since $w_{1}$ is an algebraic, then $\forall n \in \mathbb{N} \forall n \in \mathbb{N}, w_{1}^{n}$ a is also an algebraic. Let $P \in \mathbb{F}_{a}[X][Y]$ be the minimal polynomial of $w_{1}^{n}$. Now consider the embedding ${ }_{i}$ of $\mathbb{F}_{q}(X) w_{1}$ into $\overline{\mathbb{F}}_{q}\left(\left(X^{-1}\right)\right)$, which fixes $\mathbb{F}_{q}(X)$ and maps $w_{1}$ to $w_{i}$

$$
P\left(w_{i}^{n}\right)=P\left(\left(\sigma_{i}\left(w_{1}\right)^{n}\right)\right)=P\left(\sigma_{i}\left(w_{1}^{n}\right)\right)=\sigma_{i}\left(P\left(w_{1}^{n}\right)\right)=\sigma_{i}(0)=0
$$

So for all $i \leq d \quad w_{i}^{n} \quad$ satisfies $P(Y)=0 \quad$ We have, $\left[\mathbb{F}_{q}(X)\left(w_{1}^{n}\right): \mathbb{F}_{q}(X)\right] \leq\left[\mathbb{F}_{q}(X)\left(w_{1}\right): \mathbb{F}_{q}(X)\right]$. This shows that $\operatorname{deg}(P) \leq \operatorname{deg}(M)$ So $w_{1}^{n}, w_{2}^{n}, \cdots, w_{d}^{n}$ are all the roots of $P$

If $k+1 \leq i \leq d$ then $\left|w_{i}^{n}\right|=\left|w_{i}\right|^{n}<1$ (respectively. there are at least $k+1 \leq j \leq d$ such that $\left.\left|w_{j}^{n}\right|=\left|w_{j}\right|^{n}=1\right)$ and $\left|w_{i}^{n}\right|=\left|w_{i}\right|^{n}>1$ for $I=1 \ldots . k$

Therefore $\left(w_{1}^{n}, \cdots, w_{k}^{n}\right) \in S_{k}^{\prime}\left(\right.$ respectively. $\left.\in T_{k}^{\prime}\right)$ for all $n \mathbb{N}^{*}$

Proposition 3.2: Let $\left(w_{1}, \cdots, w_{k}\right) \in S_{k}^{\prime}$ then $\left\{w_{1}^{n}+\cdots, w_{k}^{n}\right\} \rightarrow 0$ as $n \rightarrow \infty$

Proof. Let $w_{1}$ be an Pisot series and $w_{1} . . w_{d}$ its conjugates. By the proof of theorem 3.1, for all $n \in \mathbb{N}$ a $w_{1}^{n}, \cdots, w_{k}^{n}$ are the roots of some degree $d$ irreducible polynomial, $P_{n}$ in $\mathbb{F}_{q}[X]$ Also,

$$
\operatorname{tr}\left(P_{n}\right)=\sum_{i=1}^{d} w_{i}^{n} \in \mathbb{F}_{q}[X]
$$

So $\left\{\operatorname{tr}\left(P_{n}\right)\right\}=0$ The above can be rewritten as

$$
\left\{\operatorname{tr}\left(P_{n}\right)=\sum_{i=1}^{d} w_{i}^{n}\right\}=\left\{w_{1}^{n}+\cdots+w_{k}^{n}+\sum_{i=k+1}^{d} w_{i}^{n}\right\}
$$

Since, for $k+1 \leq i \leq d$ by definition $\left|w_{i}\right|<1$ therefore $w_{i}^{n} \rightarrow 0$. Thus $\left\{\sum_{i=k+1}^{d} w_{i}^{n}\right\} \rightarrow 0$. Therefore $\left\{w_{1}^{n}+\cdots w_{k}^{n}\right\} \rightarrow 0$.

Proposition 3.3: Let $\left(w_{1}, \cdots, w_{k}\right) \in S_{k}^{\prime}$ with minimal polynomial $p \in \mathbb{F}_{q}[X][Y]$ of degree $k+1$ and $w=w_{1}, \ldots w_{k} \ldots w_{k+1}$ the conjugates of $w$ If $w$ is unit then $\frac{(-1)^{k+1}}{c} w_{1} \cdots w_{k} \in S^{\prime}$.

Proof. Let $\left(w_{1}, \cdots, w_{k}\right) \in S_{k}^{\prime}$ with minimal polynomial $P$ has degree $k+1$ and $P(0)=C$

Let $w_{k+1}$ be the $k+1$ root of $P$ Since

$$
P(Y)=\left(Y-w_{1}\right)\left(Y-w_{2}\right) \cdots\left(Y-w_{k+1}\right) \text {. }
$$

Consider

$\frac{1}{w_{1}}, \frac{1}{w_{2}}, \cdots, \frac{1}{w_{k+1}}=\frac{(-1)^{k+1}}{c} w_{1} \cdots w_{k}$.

Clearly $Q$ is unit, irreducible over $\mathbb{F}_{q}[X][Y]$. and has roots $\frac{1}{w_{1}}, \frac{1}{w_{2}}, \cdots, \frac{1}{w_{k+1}}=\frac{(-1)^{k+1}}{c} w_{1} \cdots w_{k}$. We have $\left|\frac{1}{w_{k+1}}\right|=\left|\frac{(-1)^{k+1} w_{1} \cdots w_{k}}{c}\right|=\left|w_{1} \cdots w_{k}\right|>1$ and $\left|\frac{1}{w_{i}}\right|<1$ for $i=1,2, \ldots k$ Therefore $(-1)^{k+1} w_{1} \cdots w_{k}$ is a Pisot series.

\section{Formal Pisot-k pairs (respectively Salem) Series}

Theorem 3.4: Let the polynomial

$$
P(Y)=A_{n} Y^{n}+A_{n-1} Y^{n-1}+A_{n-2} Y^{n-2}+\cdots+A_{1} Y+A_{0}, A_{0} \neq 0, A_{i} \in \mathbb{F}_{q}[X] .
$$

$P$ has exactly $k$ roots that lie outside the unit disc sauch that one the these roots are the biggest and all remaining roots have modulus strictly less than 1(respectively the other roots are have modulus inferior or equal to 1 and at least exist a root of module equals to 1 ) if and only if $\left|A_{n-k}\right|>\sup _{i \neq n-k}\left|A_{i}\right|$. respectively $\left|A_{n-k}\right|>\sup _{i \neq n-k}\left|A_{i}\right|$ and $\left|A_{n-k}\right|=\sup _{i<n-k}\left|A_{i}\right|$.)

Proof. Let $w=w_{1}, \ldots w_{2} \ldots w_{n}$ be the roots of $P(Y)$ such that 
$\left|w_{1}\right|>\left|w_{2}\right| \geq \cdots \geq\left|w_{k}\right|>1>\left|w_{k+1}\right| \geq \cdots \geq\left|w_{n}\right|$.

We have

$$
\begin{cases}\left|\sum_{1 \leq i \leq n} w_{i}\right|=\left|\frac{A_{n-1}}{A_{n}}\right| \leq\left|w_{1}\right| & <\left|w_{1} \cdots w_{k}\right|=\left|\frac{A_{n-k}}{A_{n}}\right| \\ \left|\sum_{1 \leq i_{1} \leq i_{2} \leq n} w_{i_{1}} w_{i_{2}}\right|=\left|\frac{A_{n-2}}{A_{n}}\right| \leq\left|w_{1} w_{2}\right| & <\left|w_{1} \cdots w_{k}\right|=\left|\frac{A_{n-k}}{A_{n}}\right| \\ \vdots & \vdots \\ \left|\sum_{1 \leq i_{1}<i_{2} \leq \cdots \leq i_{k-1} \leq n} w_{i_{1}} w_{i_{2}} \cdots w_{i_{k-1}}\right|=\left|\frac{A_{n-k+1}}{A_{n}}\right| \leq\left|w_{1} \cdots w_{k-1}\right| & <\left|w_{1} \cdots w_{k}\right|=\left|\frac{A_{n-k}}{A_{n}}\right| \\ \left|\sum_{1 \leq i_{1}<i_{2} \leq \cdots \leq i_{k+1} \leq n} w_{i_{1}} w_{i_{2}} \cdots w_{i_{k+1}}\right|=\left|\frac{A_{n-k-1}}{A_{n}}\right| \leq\left|w_{1} \cdots w_{k+1}\right| & <\left|w_{1} \cdots w_{k}\right|=\left|\frac{A_{n-k}}{A_{n}}\right| \\ \vdots & \vdots \\ \left|\prod_{1 \leq i \leq n} w_{i}\right|=\left|\frac{A_{0}}{A_{n}}\right|=\left|w \cdots w_{n}\right| & <\left|w \cdots w_{k}\right|=\mid \frac{A_{n-k} \mid}{A_{n}}\end{cases}
$$

Then

$$
\left|A_{n-k}\right|>\sup _{i \neq n-k}\left|A_{i}\right| \text {. }
$$

Second, Prove the sufficiency by the symmetrical relations of the roots of a polynomial. Let $w=w_{1}, \ldots w_{2} \ldots w_{n}$ be the roots of $P(Y)$ such that $\left|w_{1}\right|>\left|w_{2}\right| \geq\left|w_{3}\right| \geq \cdots \geq\left|w_{n}\right|$. Then

$$
\left|\frac{A_{n-k+1}}{A_{n}}\right|=\left|\sum_{1 \leq i_{1}<i_{2} \leq \cdots \leq i_{k-1} \leq n} w_{i_{1}} w_{i_{2}} \cdots w_{i_{k-1}}\right|=\left|w_{1} \cdots w_{k-1}\right|<\left|\frac{A_{n-k}}{A_{n}}\right|=\left|w_{1} \cdots w_{k}\right| \text {. }
$$

So

$\left|w_{k}\right|>1$ and $\left|w_{1}\right|>\left|w_{2}\right| \geq\left|w_{3}\right| \geq \cdots \geq\left|w_{k}\right|>1$.

on the other hand

$$
\left|\frac{A_{n-k-1}}{A_{n}}\right|=\left|\sum_{1 \leq i_{1}<i_{2} \leq \cdots \leq i_{k+1} \leq n} w_{i_{1}} w_{i_{2}} \cdots w_{i_{k+1}}\right|=\left|w_{1} \cdots w_{k+1}\right|<\left|\frac{A_{n-k}}{A_{n}}\right|=\left|w_{1} \cdots w_{k}\right| \text {. }
$$

Then

$$
\left|w_{k+1}\right|<1 \text { and } 1>\left|w_{k+1}\right|>\left|w_{k+2}\right| \geq \cdots \geq\left|w_{n}\right| \text {. }
$$

Now, if $\left|A_{n-k}\right|=\sup _{i<n-k}\left|A_{i}\right|$.

Let $w=w_{1}, \ldots w_{2} \ldots w_{k}$ be the roots of $P(Y)$ such that

$\left|w_{1}\right|>\left|w_{2}\right| \geq \cdots \geq\left|w_{k}\right|>1>\left|w_{k+1}\right| \geq \cdots \geq\left|w_{n}\right|$ and $\exists k+1 \leq j \leq n$ such that $\left|w_{j}\right|=1$.

We have

$$
\begin{cases}\left|\sum_{1 \leq i \leq n} w_{i}\right|=\left|\frac{A_{n-1}}{A_{n}}\right| \leq\left|w_{1}\right| & <\left|w_{1} \cdots w_{k}\right|=\left|\frac{A_{n-k}}{A_{n}}\right| \\ \left|\sum_{1 \leq i_{1}<i_{2} \leq n} w_{i_{1}} w_{i_{2}}\right|=\left|\frac{A_{n-2}}{A_{n}}\right| \leq\left|w_{1} w_{2}\right| & <\left|w_{1} \cdots w_{k}\right|=\left|\frac{A_{n-k}}{A_{n}}\right| \\ \vdots & \vdots \\ \left|\sum_{1 \leq i_{1}<i_{2} \leq \cdots \leq i_{k-1} \leq n} w_{i_{1}} w_{i_{2}} \cdots w_{i_{k-1}}\right|=\left|\frac{A_{n-k+1}}{A_{n}}\right| \leq\left|w_{1} \cdots w_{k-1}\right| & <\left|w_{1} \cdots w_{k}\right|=\left|\frac{A_{n-k}}{A_{n}}\right| \\ \left|\sum_{1 \leq i_{1}<i_{2} \leq \cdots \leq i_{k+1} \leq n} w_{i_{1}} w_{i_{2}} \cdots w_{i_{k+1}}\right|=\left|\frac{A_{n-k-1}}{A_{n}}\right| \leq\left|w_{1} \cdots w_{k+1}\right| & \leq\left|w_{1} \cdots w_{k}\right|=\left|\frac{A_{n-k}}{A_{n}}\right| \\ \vdots \sum_{1 \leq i_{1}<i_{2} \leq \cdots \leq i_{j} \leq n} w_{i_{1}} w_{i_{2}} \cdots w_{i_{j}}|=| \frac{A_{n-(k+j)}}{A_{n}}|\leq| w_{1} \cdots w_{k-j} \mid & \leq\left|w_{1} \cdots w_{k}\right|=\left|\frac{A_{n-k}}{A_{n}}\right| \\ \vdots & \vdots \\ \left|\prod_{1 \leq i \leq n} w_{i}\right|=\left|\frac{A_{0}}{A_{n}}\right|=\left|w \cdots w_{n}\right| & \leq\left|w \cdots w_{k}\right|=\left|\frac{A_{n-k}}{A_{n}}\right|\end{cases}
$$

$$
\begin{aligned}
& \text { Then } \\
& \left|A_{n-k}\right|>\sup _{i>n-k}\left|A_{i}\right| \text { and }\left|A_{n-k}\right|=\sup _{i<n-k}\left|A_{i}\right| \text {. }
\end{aligned}
$$

Consequence 3.5: Let the polynomial

$$
P(Y)=A_{n} Y^{n}+A_{n-1} Y^{n-1}+A_{n-2} Y^{n-2}+\cdots+A_{1} Y+A_{0}, A_{0} \neq 0, A_{i} \in \mathbb{F}_{q}[X] .
$$

$P$ has exactly $k$ roots that lie outside the unit disc sauch that one the these roots are the biggest and all remaining roots have modulus strictly less than 1(respectively the other roots are have modulus inferior or equal to 1 and at least exist a root of module equals to 1) if and only if $\left|A_{n-k}\right|>\left|A_{n-k+1}\right|>\cdots>\left|A_{n-1}\right|>\sup _{i<n-k}\left|A_{i}\right|$. respectively $\left|A_{n-k}\right|>\sup _{i \neq n-k}\left|A_{i}\right|$ and $\left|A_{n-k}\right|=\sup _{i<n-k}\left|A_{i}\right|$.)

\section{Corollary 3.6: Let $P$ be the polynomial}

$$
\Lambda(Y)=\lambda_{n} Y^{n}+\lambda_{n-1} Y^{n-1}+\lambda_{n-2} Y^{n-2}+\cdots+\lambda_{1} Y+\lambda_{0}, \lambda_{0} \neq 0, \lambda_{i} \in \mathbb{F}_{q}[X]
$$

such that $\left|\lambda_{n-k}\right|>\sup _{i \neq n-k}\left|\lambda_{i}\right|$. If $\left|\lambda_{n-k}\right| \geq\left|\lambda_{n-k+1}\right|\left|\lambda_{n-1}\right|$, then $\Lambda$ has no roots in $\mathbb{F}_{q}\left(\left(X^{-1}\right)\right)$

Proof. By the previous Theorem $\Lambda$ has $k$ roots of modules $>1$ with a different value and the other roots are of modules $<1$ Let $w=w_{1}, \ldots w_{2} \ldots$. $w_{n}$ be the roots of $P(Y)$ such that

$\left|w_{1}\right|>\left|w_{2}\right| \geq \cdots \geq\left|w_{k}\right|>1>\left|w_{k+1}\right| \geq \cdots \geq\left|w_{n}\right|$.

We have

$$
\left|\lambda_{n-k}\right| \geq\left|\lambda_{n-k+1}\right|\left|\lambda_{n-1}\right| \text {. }
$$

And so

$\left|w_{1} \cdots w_{k}\right| \geq\left|w_{1} \cdots w_{k-1}\right|\left|w_{1}\right|$.

This gives us that

$\left|w_{k}\right| \geq\left|w_{1}\right|$.

witch is absurd. So $\Lambda$ is irreducible on $\mathbb{F}_{q}[X][Y]$

Theorem 3.7: Let $\left(w_{i}\right) \in \mathbb{F}_{q}\left(\left(X^{-1}\right)\right) \quad$ for $\quad i=1, \cdots, k \quad$ witch $\left|w_{1}\right|>\left|w_{2}\right| \geq \cdots\left|w_{k}\right|>1$ be the are roots of the polynomial

$\Lambda(Y)=Y^{n}+\lambda_{n-1} Y^{n-1}+A_{n-2} Y^{n-2}+\cdots+\lambda_{1} Y+\lambda_{0}, \lambda_{i} \in \mathbb{F}_{q}[X], \lambda_{0} \neq 0$ and $\left|\lambda_{n-2}\right|>\sup _{i \neq n-2}\left|\lambda_{i}\right|$

If $\operatorname{deg} \lambda_{n-k}=\operatorname{deg} \lambda_{n-k+1}+\operatorname{deg} \lambda_{n-1}$, then $\Lambda$ is the minimal polynomial of $w$ and $\left(w=w_{1}, \ldots w_{k}\right) \in S_{k}$

Proof. By the Theorem 3.4, $P$ has exactly $k$ conjugates of $w=w_{1}, \ldots w_{k}$ that lie outside the unit disc and all remaining conjugates have modulus strictly less than $<1$ Let's $w_{i}$ for $i=k+1, \ldots n$ witch $\left|w_{i}\right|<1$ the authors roots of $P$. Show that $\Lambda(Y)$ is irreducible.

By the condition of the Theorem, $\Lambda(0)=\lambda_{0} \neq 0$ hence, all roots of the polynomial $\Lambda(Y)$. are not equal to 0

Let $\Lambda(Y)=\Lambda_{1}(Y) . \Lambda_{2}(Y)$ where $\Lambda_{i}(Y), i=1,2$, has of the coefficients in $\mathbb{F}_{q}[X]$.

Suppose in the first $w_{1}, \ldots w_{k}$ are the roots of $\Lambda_{1}$ and the other roots are of $\Lambda_{2}$ Clearly, the absolute value of leading coefficient of the polynomial $\Lambda_{2}$ superior or equal 1 with is absurd because $\Lambda_{2}$ has only roots $w_{i}$ such that

$0<\left|w_{i}\right|<1$

Suppose in the second that $\Lambda_{1}$ is the polynomial of the series $w_{1}, \ldots w_{k^{\prime}}$ and $\Lambda_{2}$ the polynomial of the series $w_{k^{\prime}+1} \ldots w_{k^{\prime \prime}}$ such that $k^{\prime}+k^{\prime \prime}=k$

$$
\begin{aligned}
\Lambda(Y)= & \Lambda_{1}(Y) \cdot \Lambda_{2}(Y) \\
= & \left(Y^{s}+A_{s-1} Y^{s-1}+\cdots+A_{s-k^{-}} Y^{s-k^{\prime}}+\cdots+A_{1} Y+A_{0}\right)\left(Y^{m}+B_{m-1} Y^{m-1}+\cdots\right. \\
& \left.+B_{m-k^{\prime \prime}}{ }^{m-k^{\prime \prime}}+\cdots+B_{1} Y+B_{0}\right)
\end{aligned}
$$


Then we have

$$
\begin{cases}\lambda_{n-1}= & A_{s-1}+B_{m-1} \\ \lambda_{n-k+1}= & \cdots+A_{s-k^{\prime}+1} B_{m-k^{\prime \prime}}+A_{s-k^{\prime}} B_{m-k^{\prime \prime}+1}+\cdots \\ \lambda_{n-k}= & \cdots+A_{s-k^{\prime}} B_{m-k^{\prime \prime}}+\cdots\end{cases}
$$

This gives us that

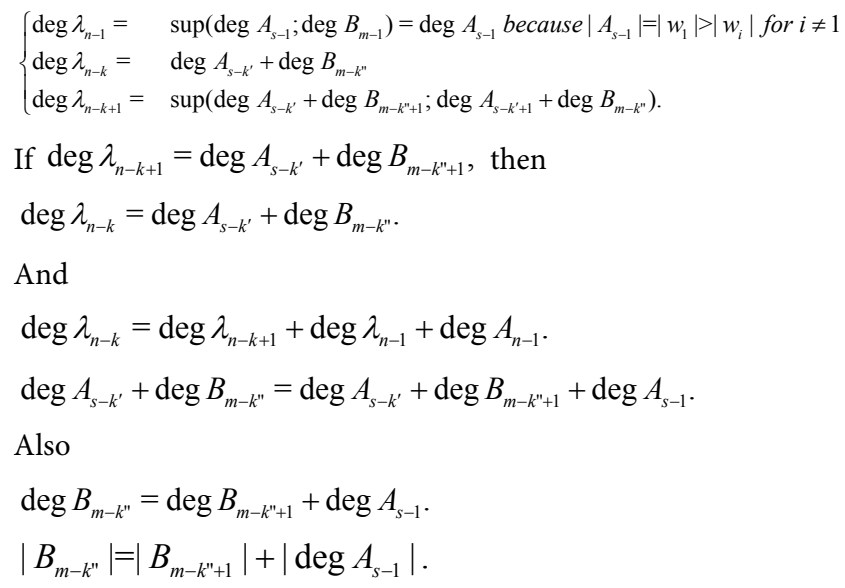

This gives us that

$\left|w_{k^{\prime}+1} \cdots w_{k}^{\prime \prime}\right|=\left|w_{k^{\prime}+1} \cdots w_{m-k^{\prime \prime}+1}\right|\left|w_{1}\right|$.

Therefore

$\left|w_{k}^{\prime \prime}\right|=\left|w_{1}\right|$.

With is absurd.

Now if $\operatorname{deg} \lambda_{n-k+1}=\operatorname{deg} A_{s-k^{\prime}+1}+\operatorname{deg} B_{m-k^{n}}$, then

$\operatorname{deg} \lambda_{n-k}=\operatorname{deg} A_{s-k^{\prime}}+\operatorname{deg} B_{m-k^{\prime \prime}}$

And

$\operatorname{deg} \lambda_{n-k}=\operatorname{deg} \lambda_{n-k+1}+\operatorname{deg} \lambda_{n-1}+\operatorname{deg} A_{n-1}$.

$\operatorname{deg} A_{s-k^{\prime}}+\operatorname{deg} B_{m-k^{\prime \prime}}=\operatorname{deg} A_{s-k^{\prime}+1}+\operatorname{deg} B_{m-k^{\prime \prime}}+\operatorname{deg} A_{s-1}$.

This gives us that

$$
\begin{aligned}
& \left|A_{s-k^{\prime}}\right|=\left|A_{s-k^{\prime}+1}\right| .\left|\operatorname{deg} A_{s-1}\right| . \\
& \left|A_{s-k^{\prime}}\right|=\left|A_{s-k^{\prime}+1}\right| .\left|\operatorname{deg} A_{s-1}\right| .
\end{aligned}
$$

We obtain

$$
\left|w_{1} \cdots w_{k^{\prime}}\right|=\left|w_{1} \cdots w_{k^{\prime}-1}\right|\left|w_{1}\right|
$$

Therefore

$$
\left|w_{k^{\prime}}\right|=\left|w_{1}\right| \text {. }
$$

With is absurd. $w_{1}, \ldots w_{k^{\prime}+1} \ldots w_{k} "$

Let $\Lambda(Y)=$ where $\Lambda_{i}(Y), i=1,2,3$ has of the coefficients in $\mathbb{F}_{q}[X]$. Suppose in the first $w_{1}, \ldots w_{k}$ are the roots of $\Lambda_{1} w_{k^{\prime}+1} \ldots w_{k}$ " are the roots of $\Lambda_{2}$, such that $k^{\prime}+k^{\prime \prime}=k$ and the other roots are of $\Lambda_{3}$. Clearly, the absolute value of leading coefficient of the polynomial $\Lambda_{3}$ superior or equal 1 with is absurd because $\Lambda_{2}$ has only roots $w_{i}$ such that $0<\left|w_{i}\right|<1$.

We conclude that $\Lambda$ is the minimal polynomial of and $\left(w_{1} \ldots w_{k}\right) \in S_{k}$

Corollary 3.8: Let $\mathbb{P}_{k}(n, h)$ the set of the element of Pisot- $k$ series in ${ }_{q}\left(\left(X^{-1}\right)\right)$ of degree $n$ and of height logarithmic $h$ Then the numbers of the elements of $\mathbb{P}_{k}(n, h)$ are

$$
\left|\mathcal{P}_{k}(n, h)\right| \leq(q-1)\left(q^{h-1}-1\right) q^{n(h-1)-1} .
$$
$(n, h)$ is

Proof. The set of the minimal polynomials of the elements of $\mathbb{P}_{k}$

$$
\mathcal{L}_{k}(n, h)=\left\{P(Y)=Y^{n}+A_{n-1} Y^{n-1}+\cdots+A_{n-k} Y^{n-k}+\cdots+A_{0}:\right.
$$

$h=\operatorname{deg} A_{n-k}>\sup \operatorname{deg}_{i \neq n-k} A_{i}$ and $\operatorname{deg} A_{n-k}=\operatorname{deg} A_{n-k+1}+\operatorname{deg} A_{n-1}$.

By the consequence 3.5, we have $h=\left|A_{n-k}\right|>\left|A_{n-k+1}\right|>\cdots>\left|A_{n-1}\right|>\sup _{i<n-k}\left|A_{i}\right|$. we obtain

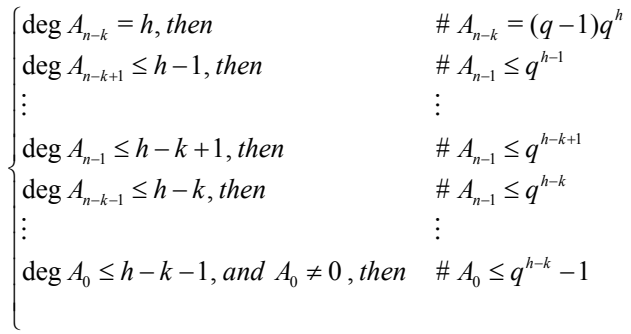

Therefore

$$
\begin{aligned}
& \left|\mathcal{L}_{k}(n, h)\right| \\
& \leq(q-1) q^{h} \cdot q^{h-1} \cdots q^{h-k+1} q^{(h-k)(n-k+1)}\left(q^{h-k}-1\right) \\
& \leq(q-1)\left(q^{h-1}-1\right) q^{(h-k)(n-k+1)}\left(q^{h-k}-1\right) q^{[h+(h-1)+\cdots(h-k+1)]} \\
& \leq(q-1)\left(q^{h-1}-1\right) q^{(h-k)(n-k+1)}\left(q^{h-k}-1\right) q^{\frac{k}{2}(2 h-k+1)} .
\end{aligned}
$$

\section{Conclusion}

The presentation and estimation for the number of the Pisot-k power formal series, precisely we will give their degrees and their logarithmic heights.

\section{Acknowledgements}

I would like to thank Dr. Mabrouk ben ammar and Amara chandoul for all of his guidance and instruction, and for introducing me to the study of Pisot numbers.

\section{References}

1. Brauer A (1951) On algebraic equations with all but one root in the interior of the unit circle. Math Nachr 4: 250-257.

2. Akiyama S (2006) Positive finiteness of number systems. Number theory, Tradition and Modernization.

3. Bateman P, Duquette AL (1962) The analogue of Pisot-Vijayaraghavan numbers in fields of power series. III J Math 6: 594-406.

4. Chandoul A, Jellali M, Mkaouar M (2011) Irreducibility criterion over finite fields. Communication in Algebra 39: 3133-3137.

5. Chandoul A, Jellali, Mkaouar M (2013) The smallist Pisot element over $\mathbb{F}_{q}\left(\left(X^{-1}\right)\right)$, Communication in Algebra 56: 258-264.

6. Sprindzuk VG (1963) Mahler's problem in metric number theory, Translaion of Mathematical monographs. Amer math Soc.

Citation: Kthiri H (2016) Pisot-K Elements in the Field of Formal Power Series over Finite Field. J Phys Math 7: 194. doi: 10.4172/2090-0902.1000194 